\title{
I processi inclusivi nella prima infanzia tra diritti e responsabilità
}

\author{
Roberta Caldin
}

\begin{abstract}
In this paper, we address some issues regarding inclusive education, in particular in nursery schools. Some of its parts clearly refer to indications contained in the Convention on the Rights of Persons with Disabilities and the World Report on Disability relating to the access to education of boys and girls with disabilities, in the world.

The facilitative contexts, the meeting with the parents and the educational and helpful relationships are some essential elements of the educational aspect of a nursery school: these also open the discussion on the presence of parental elements in the educational help experts and on the educational care.
\end{abstract}

Keywords: UN Convention on the Rights of Persons with Disabilities; National Observatory on the Status of Persons with Disabilities; educational services; childhood.

Nel presente contributo si affrontano alcuni temi dell'educazione inclusiva che riguardano, soprattutto, l'asilo nido.

Vengono richiamate alcune indicazioni dalla Convention on the Rights of Persons with Disabilities e dal World Report on Disability, concernenti l'accesso all'istruzione e all'educazione dei bambini e delle bambine disabili, nel mondo.

I contesti facilitanti, l'incontro con i genitori e la relazione educativa e di aiuto costituiscono alcuni degli elementi irrinunciabili della cifra educativa di un asilo nido: questi aprono anche alla disamina sulla presenza di elementi genitoriali nelle professioni educative di aiuto e sulla "cura" educativa.

Parole chiave: Convenzione delle Nazioni Unite sui diritti delle persone disabili; Osservatorio Nazionale sulla condizione delle persone con disabilità; servizi educativi; infanzia.

\footnotetext{
${ }^{1}$ Docente di Pedagogia Speciale - Dipartimento di Scienze dell'Educazione - Università di Bologna.
} 


\section{La Convenzione delle Nazioni Unite sui diritti delle Persone con Disabilità e l'Osservatorio Nazionale sulla condizione delle Persone con disabilità}

Con la Legge n. 18 del 3 marzo 2009², l'Italia si assume un rilevante impegno, di fronte alla comunità internazionale, ratificando la Convenzione delle Nazioni Unite sui Diritti delle Persone con Disabilità ${ }^{3}$. Con i suoi 50 articoli, la Convenzione ONU del 2006 rappresenta il primo grande trattato sui diritti umani del nuovo millennio: è un documento di grandissima importanza per la promozione di una nuova cultura sulla condizione delle persone con disabilità e delle loro famiglie.

La Convenzione è frutto di un lavoro ampio e collegiale, che considera la disabilità un concetto in evoluzione $\mathrm{e}$ in interazione con il contesto nel quale ci si trova a vivere; prende atto che le persone con disabilità continuano a incontrare ostacoli nella propria partecipazione (come membri della società) e ritiene che solo la partecipazione possa dare un accresciuto senso di appartenenza e di significatività nella progressione dello sviluppo umano, sociale ed economico. In particolare, la Convenzione riconosce l'importanza, per le persone con disabilità, della loro autonomia ed indipendenza, compresa la libertà di compiere scelte autonome; riconosce che i bambini con disabilità dovrebbero poter godere pienamente di tutti i diritti umani e delle libertà fondamentali - su base di eguaglianza rispetto agli altri bambini sottolineando l'accessibilità dell'ambiente fisico, sociale, economico e culturale, il diritto alla salute, all'istruzione, all'informazione e alla comunicazione; per la Convenzione, la famiglia è il naturale e fondamentale nucleo della società e merita la protezione da parte della società e dello Stato.

L'Italia ratifica e rende esecutiva la Convenzione delle Nazioni Unite sui Diritti delle Persone con Disabilità e, con il medesimo provvedimento (art. 3), istituisce l'Osservatorio Nazionale sulla Condizione delle Persone con Disabilità ${ }^{4}$ : tale Osservatorio (OND) è, infatti, un organismo collegiale presieduto dal Ministro del Lavoro e delle Politiche Sociali; è composto da 40 membri effettivi e da un numero massimo di 10 invitati permanenti, in rappresentanza delle diverse amministrazioni pubbliche e del mondo dell'associazionismo, rappresentativo delle persone con disabilità. L'Osservatorio, nel suo secondo mandato - 20142016 - si avvale del supporto di una Segreteria Tecnica costituita nell'ambito

${ }^{2}$ http://www.parlamento.it/parlam/leggi/09018l.htm. Consultato il 9 dicembre 2016.

${ }^{3}$ Convention on the Rights of Persons with Disabilities http://www.un.org/disabilities/ convention/conventionfull.shtml. Prepared by the UN Web Services Section, Department of Public Information (c) United Nations 2006. Consultato il 9 dicembre 2016.

${ }^{4} \mathrm{http}: / / \mathrm{ww}$ w.lavoro.gov.it/temi-e-priorita/disabilita-e-non-autosufficienza/focus-on/ Osservatorio/Pagine/default.aspx. Consultato il 9 dicembre 2016. 
delle ordinarie risorse umane e strumentali della Direzione Generale per l'inclusione e le politiche sociali del Ministero5. L'OND ha funzioni consultive e di supporto tecnico-scientifico per l'elaborazione delle politiche nazionali in materia di disabilità, con particolare riferimento alla:

- promozione dell'attuazione della Convenzione sui Diritti delle Persone con Disabilità e all'elaborazione di un rapporto dettagliato sulle misure adottate (di cui all'articolo 35 della stessa Convenzione), in raccordo con il Comitato interministeriale dei diritti umani;

- predisposizione di un Programma di Azione biennale per la promozione dei diritti e l'integrazione delle persone con disabilità, in attuazione della legislazione nazionale e internazionale;

- raccolta di dati statistici che illustrino la condizione delle persone con disabilità, anche con riferimento alle diverse situazioni territoriali;

- realizzazione di studi e ricerche che possano contribuire ad individuare aree prioritarie verso cui indirizzare azioni e interventi per la promozione dei diritti delle persone con disabilità 6 .

All'interno dell'Osservatorio è istituito un Comitato Tecnico-Scientifico con finalità di analisi ed indirizzo scientifico in relazione alle attività e ai compiti dell'Osservatorio. Il Comitato è composto da un rappresentante del Ministero del Lavoro e del Ministero della Salute, un rappresentante delle Regioni e uno delle autonomie locali, due rappresentanti delle associazioni nazionali maggiormente rappresentative delle persone con disabilità e tre esperti facenti parte dell'Osservatorio ${ }^{7}$.

Con il Decreto del Presidente della Repubblica del 4 ottobre $2013^{8}$, l'Italia adotta il I Programma di Azione Biennale per la promozione dei diritti e l'integrazione delle persone con disabilità, aprendo un nuovo scenario di riferimento politico e programmatico dato che, finalmente, la condizione di disabilità di tanti cittadini italiani viene rappresentata per il suo valore e come un'irrinunciabile area di tutela dei diritti che riguarda e attraversa la politica e le istituzioni, in tutte le loro articolazioni. Il concetto fondamentale su cui si fonda il Programma di Azione biennale è quello del passaggio da

\footnotetext{
${ }^{5}$ A questo link è possibile consultare la composizione dell'OND; all'art. 3 dello stesso documento è possibile leggere anche i nominativi dei membri che compongono il Comitato Tecnico Scientifico dell'OND, tra cui quello della scrivente http://www.lavoro.gov.it/temi-epriorita/disabilita-e-non-autosufficienza/focus-on/Osservatorio/Documents/Decreto\%20 ministeriale\%20del\%204\%20luglio\%202014.pdf.

${ }^{6} \mathrm{http}: / /$ sitiarcheologici.lavoro.gov.it/AreaSociale/Disabilita/Osservatorio/Pages/default.aspx. Consultato il 9 dicembre 2016.

${ }^{7}$ L'attività dell'Organismo è normata da un Regolamento interno approvato in data 4 novembre 2014 http://sitiarcheologici.lavoro.gov.it/AreaSociale/Disabilita/Osservatorio/ Documents/Decreto_Costituzione_Osservatorio_luglio14.pdf. Consultato il 9 dicembre 2016.

${ }^{8}$ http://www.gazzettaufficiale.it/eli/id/2013/12/28/13A10469/sg.
} 
una visione delle persone con disabilità "come malate e minorate" ad una visione bio-psico-sociale basata sul rispetto dei diritti umani, valorizzante tutte le diversità - di genere, di orientamento sessuale, di cultura, di lingua, di condizione psico-fisica ecc. - e avente come focus imprescindibile il fatto che la condizione di disabilità è un intreccio tra le caratteristiche delle persone e le/ gli modalità/approcci attraverso le/i quali la società tutta organizza l'accesso ed il godimento di diritti, beni e servizi.

Tra i principi a cui si ispira, oggi, il II Programma di Azione biennale, che procede con una logica pragmatica, una prospettiva progressiva e una mediolunga temporalità, ricordiamo:

a) il rispetto per la dignità intrinseca, l'autonomia individuale, compresa la libertà di compiere le proprie scelte, e l'indipendenza delle persone;

b) la non discriminazione;

c) la piena ed effettiva partecipazione e inclusione nella società;

d) il rispetto per la differenza e l'accettazione delle persone con disabilità come parte della

diversità umana e dell'umanità stessa;

e) la parità di opportunità;

f) l'accessibilità;

g) la parità tra uomini e donne;

h) il rispetto dello sviluppo delle capacità dei minori con disabilità e il rispetto del diritto dei minori con disabilità a preservare la propria identità.

Dal punto di vista operativo, il $I I^{\wedge}$ Programma di Azione biennale può giovarsi di un'imponente mole di documenti, sviluppata in tre anni di lavoro dall'OND, che approfondisce e dettaglia le azioni programmatiche indicate. La prima bozza del II Programma di Azione biennale - approvata nel Luglio 2016 - è consultabile al link http://www.lavoro.gov.it/notizie/Documents/PDA_ Disabilita_2016_Agosto2016.pdf e si intitola Proposta di II Programma di Azione biennale per la promozione dei diritti e l'integrazione delle persone con disabilità, in attuazione della legislazione nazionale e internazionale, ai sensi dell'art. 5, co. 3, della legge 3 marzo 2009, n. 18. All'interno dell'OND sono stati istituiti 8 gruppi di lavoro'; per quanto riguarda il Gruppo 4, Processi formativi ed inclusione scolastica, i co-coordinatori sono stati Donata

\footnotetext{
${ }^{9}$ Gruppi di Lavoro dell'OND, periodo 2014-2016: Gruppo 1: Riconoscimento/certificazione della condizione di disabilità e valutazione multidimensionale finalizzata a sostenere il sistema di accesso e la progettazione personalizzata; Gruppo 2: Politiche, servizi e modelli organizzativi per la vita indipendente e l'inclusione nella società; Gruppo3: Salute, diritto alla vita, abilitazione e riabilitazione; Gruppo 4: Processi formativi ed inclusione scolastica; Gruppo 5: Lavoro e occupazione; Gruppo 6: Promozione e attuazione dei principi di accessibilità e mobilità; Gruppo 7: Cooperazione internazionale; Gruppo 8: Sviluppo del sistema statistico e di reporting sull'attuazione delle politiche.
} 
Pagetti Vivanti, Roberta Caldin, Raffaele Ciambrone. In questo contributo, presenteremo - nella prima parte - alcune sintetiche indicazioni ricavate anche dal lavoro svolto da questo Gruppo.

\section{- L'educazione della prima infanzia tra riferimenti internazionali e nazionali $^{10}$.}

L'importanza dell'attenzione che va riservata alla prima infanzia, compresa quella in situazione di disabilità, è sostenuta nella Dichiarazione Mondiale sull'Educazione per tutti, svoltasi a Jomtien, Thailand, nel 1990, e nella Convenzione sui Diritti del Fanciullo del $1989^{11}$. Quest'ultima stabilisce che "I bambini hanno diritto alla vita e alle migliori opportunità possibili di sviluppare pienamente le proprie potenzialità (art. 6), e che 'I bambini con disabilità devono essere aiutati a raggiungere il più alto livello d'indipendenza possibile e ad acquisire le capacità per partecipare pienamente e attivamente alle attività della vita quotidiana' (art. 23). Anche la Dichiarazione di Salamanca, adottata dalla Conferenza Mondiale sull'Educazione Speciale nel $1994^{12}$, identifica l'educazione precoce come area rilevantissima: la prima infanzia è fase cruciale per la scoperta "positiva" delle diversità nella condizione esistenziale, attraverso la condivisione delle attività di gioco e di apprendimento che i bambini possono svolgere. Questi programmi insistono sull'inclusione precoce e la indicano come un fattore straordinario di contenimento all'aggravamento di un deficit: l'educazione precoce, infatti, si configura come uno dei maggiori fattori di prevenzione degli elementi handicappanti aggiuntivi.

Anche il World Report on Disability della World Health Organization e della The World Bank, presentato a New York nel giugno 2011 (WHO, The World Bank, 2011) si colloca su questa linea. Come si evince dal Report - a cui rinviamo per il dettaglio, interessantissimo, dei dati e delle indicazioni i bambini e le bambine con disabilità hanno, in generale, meno probabilità di accedere ai servizi educativi e alla scuola dei loro coetanei non disabili. Secondo un'inchiesta condotta in 14 Paesi, la differenza di percentuale di bambini disabili e non disabili frequentanti la scuola primaria va dal $10 \%$

${ }^{10}$ Alcune indicazioni che qui vengono presentate si trovano anche nei documenti di lavoro del Gruppo 4 dell'OND (Co-coordinatori del gruppo di lavoro: Donata Pagetti Vivanti, Roberta Caldin, Raffaele Ciambrone).

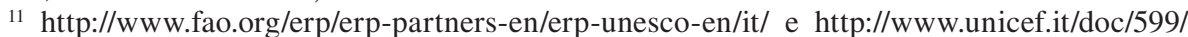
convenzione-diritti-infanzia-adolescenza.htm. Consultati il 9 dicembre 2016.

${ }^{12}$ The Salamanca Framework for Action, Article 53 http://www.unesco.org/education/pdf/ SALAMA_E.PDF Si veda anche http://www.gruppocrc.net/Salamanca-grande-successo-dellaConferenza-Mondiale-sull-Educazion. Consultati il 9 dicembre 2016. 
in India al $60 \%$ in Indonesia; a livello di scuola secondaria, le differenze di frequenza vanno dal $15 \%$ in Cambogia al $58 \%$ in Indonesia; ma anche in Paesi nei quali i tassi di scolarizzazione sono elevati nella scuola primaria nell'Europa dell'Est, ad esempio - molti bambini e bambine disabili non vanno a scuola (anche se questo trend è decisamente pronunciato nei Paesi poveri); non va dimenticato, infatti, che la mancanza di educazione/istruzione in età precoce ha un'influenza significativa sull'essere/rimanere poveri in età adulta (Ivi, pp. 206 ss).

Nel Report, l'inclusione di bambini con disabilità nei sistemi educativi e scolastici è visto come ampiamente desiderabile per l'equità delle opportunità e per la garanzia dei diritti umani e si sottolinea come l'educare/istruire i bambini insieme - disabili e non disabili - rappresenti, da un punto di vista economico, una scelta con il costo più basso. Un importante studio in Mongolia dimostra che le iscrizioni di bambini con disabilità nel sistema pre-scolastico facilita quelle nella scuola primaria, che passano dal $22 \%$ al 44\%. L'identificazione precoce dei deficit e la tempestività dell'intervento riduce la necessità del livello del supporto educativo; secondo il Report, il coinvolgimento dei genitori e dei membri della famiglia, così come degli insegnanti e della comunità tutta rappresenta uno dei fattori chiave di successo dell'inclusione (Ivi, pp. 221-227) e la centralità dei sistemi educativi e scolastici viene continuamente sottolineata. Già nella Dichiarazione di Madrid del 2002 questo era stato ribadito con forza: «Le scuole devono assumere un ruolo rilevante [per] raggiungere l'obiettivo dell'istruzione per tutti, nel rispetto dei principi della piena partecipazione e dell'uguaglianza. L'istruzione ha un ruolo fondamentale nella costruzione del futuro per tutti, sia per l'individuo, sia per la persona come membro della società e del mondo del lavoro. Il sistema educativo deve essere il luogo centrale che assicuri lo sviluppo personale e l'inclusione sociale, che consentiranno ai bambini e ai giovani di essere quanto più indipendenti possibili. Il sistema educativo è il primo passo verso una società dell'integrazione $»^{13}$. Con tali precisazioni, nella Dichiarazione di Madrid si delineava non solo la centralità e la rilevanza del sistema scolastico, ma la focalizzazione sulla irrinunciabilità della funzione sociale dei sistemi educativi e scolastici, che apre la strada all'esigibilità dei diritti umani.

$\mathrm{E}$, su quest'ultima questione, il concetto di inclusione aiuta a intravedere il cammino da percorrere. Infatti, mentre con integrazione si intende una situazione di fatto che, prevalentemente, si riferisce all'ambito educativo in senso stretto, guarda al singolo alunno, interviene prima sul soggetto e poi

\footnotetext{
$13 \mathrm{http} / / / \mathrm{ww}$ w.includ-ed.eu/resource/declaration-madrid-2002-\%E2\%80\%9Cnondiscrimination-plus-positive-action-equals-social-inclusion\%E2\%80\%9D. Consultato il 9 dicembre 2016.
} 
sul contesto, incrementando una risposta specialistica, l'educazione inclusiva (Inclusive education) si riferisce alla globalità delle sfere educativa, sociale e politica, guarda a tutti/e gli/le alunni/e (indistintamente/differentemente), interviene prima sul contesto, poi sul soggetto, trasformando la risposta specialistica in ordinaria: «La pratica dell'inclusive education indica una centratura sul contesto oltreché sul bambino come possibilità e diritto per tutti i bambini/e i ragazzi/e di accedere ai percorsi scolastici ordinari e non speciali o segreganti» (UNESCO, 2005; 2006; 2010). Infatti, l'Inclusive education:

- ha una dimensione sociale: non prima "riabilitare", poi socializzare, poi far apprendere, ma integrarsi in un contesto scolastico ricco nel confronto con gli educatori, i docenti e con i compagni;

- può rendere tangibile l'appartenenza (e i processi di identità) ad un gruppo educativo, alla classe, alla comunità scolastica ecc.;

- favorisce il percorso di apprendimento interindividuale e intraindividuale (co-costruzione del sapere nell'interazione).

L'educazione inclusiva - come imperativo morale - si rifà ad un modello antropocentrico (basato sulle esigenze costitutive e di sviluppo di ciascuno), ad un modello complesso di disabilità (che contempla apprendimento e socializzazione), alla familiarizzazione e al protagonismo delle persone in situazioni problematiche e richiede la frequentazione/partecipazione attiva in contesti ad alto apprendimento simbolico (gli spazi educativi e scolastici, le classi, le aule universitarie ecc.).

Ma l'educazione inclusiva, per essere efficace, necessita anche di alcune condizioni: l'educatore (o l'insegnante) necessita di una permanenza nella professione che superi la precarietà e sviluppi delle competenze (non sostituibili) che possono rendere competente il contesto; i contesti devono favorire una pluralità di incontri con adulti significativi, che lavorino insieme e garantiscano un sistema di convivenza plurale e ricca, nel quale il ruolo dell'imitazione nei processi di apprendimento e la funzione di prevenzione costituiscano fattori di innovazione e di cambiamento sociale; se pensiamo all'asilo nido, ad esempio, è chiaro che questa dovrebbe essere la sua cifra stilistica.

Il Comitato dei ministri del Consiglio d'Europa, con la Raccomandazione del 12 dicembre $2012^{14}$, indica come tema prioritario quello del diritto all'educazione, intesa come educazione "di qualità". A parere del Comitato dei ministri, tale diritto riveste un ruolo cruciale nello sviluppo delle società

14 Committee of Ministers, Recommendation adopted on 12 December 2012, CM/Rec(2012)13, Ensuring quality education; si veda il link http://www. ohchr.org/EN/Issues/Education/Training/Compilation/Pages/26Committee ofMinistersRecommendationCMRec(2012)13tomemberStatesonensuringqualityeducation(2012). aspx. Consultato il 9 dicembre 2016. 
europee perché rappresenta anche una responsabilità pubblica degli Stati membri. L'istruzione e l'educazione di qualità, infatti, devono essere garantite a tutti: è necessario, dunque, che le Autorità pubbliche nazionali si sforzino di includere nelle loro politiche una "formazione di qualità" che investa anche i genitori dei bambini. Inoltre, il Comitato invita ad accertare con precisione le situazioni che necessitano di interventi educativi specifici, sollecitando le autorità pubbliche a trovare soluzioni opportune il più rapidamente possibile.

L'articolo 24 della Convenzione ONU sui Diritti delle Persone con Disabilità ${ }^{15}$

${ }^{15} \mathrm{http}: / /$ ec.europa.eu/social/main.jsp?catId=1138. Consultato il 9 dicembre 2016. Article 24-Education

1. States Parties recognize the right of persons with disabilities to education. With a view to realizing this right without discrimination and on the basis of equal opportunity, States Parties shall ensure an inclusive education system at all levels and life long learning directed to:

a) The full development of human potential and sense of dignity and self-worth, and the strengthening of respect for human rights, fundamental freedoms and human diversity;

b) The development by persons with disabilities of their personality, talents and creativity, as well as their mental and physical abilities, to their fullest potential;

c) Enabling persons with disabilities to participate effectively in a free society.

2. In realizing this right, States Parties shall ensure that:

a) Persons with disabilities are not excluded from the general education system on the basis of disability, and that children with disabilities are not excluded from free and compulsory primary education, or from secondary education, on the basis of disability;

b) Persons with disabilities can access an inclusive, quality and free primary education and secondary education on an equal basis with others in the communities in which they live;

c) Reasonable accommodation of the individual's requirements is provided;

d) Persons with disabilities receive the support required, within the general education system, to facilitate their effective education;

e) Effective individualized support measures are provided in environments that maximize academic and social development, consistent with the goal of full inclusion.

3. States Parties shall enable persons with disabilities to learn life and social development skills to facilitate their full and equal participation in education and as members of the community. To this end, States Parties shall take appropriate measures, including:

a) Facilitating the learning of Braille, alternative script, augmentative and alternative modes, means and formats of communication and orientation and mobility skills, and facilitating peer support and mentoring;

b) Facilitating the learning of sign language and the promotion of the linguistic identity of the deaf community;

c) Ensuring that the education of persons, and in particular children, who are blind, deaf or deafblind, is delivered in the most appropriate languages and modes and means of communication for the individual, and in environments which maximize academic and social development.

4. In order to help ensure the realization of this right, States Parties shall take appropriate measures to employ teachers, including teachers with disabilities, who are qualified in sign language and/or Braille, and to train professionals and staff who work at all levels of education. Such training shall incorporate disability awareness and the use of appropriate augmentative and alternative modes, means and formats of communication, educational techniques and materials to support persons with disabilities. 
non fa specifico riferimento all'educazione precoce, ma - imponendo agli Stati Parti di garantire l'accesso all'educazione ai bambini, ai giovani e agli adulti con disabilità, su base di uguaglianza con gli altri, in un sistema inclusivo - implicitamente comprende anche i bambini in età prescolare. Infatti, se impediti nell'accesso ai contesti educativi, i bambini con disabilità possono risultare penalizzati nel proprio sviluppo e nel proprio potenziale, con conseguenze significative sia a livello sociale che economico. Tale penalizzazione ha una ricaduta anche sulle famiglie e sulla comunità tutta: infatti, sono proprio le situazioni maggiormente problematiche a trarre i più alti benefici da una frequentazione precoce dei contesti educativi territoriali ${ }^{16}$.

Non va dimenticato, inoltre, che la presenza di un bambino con disabilità in un servizio educativo per la prima infanzia è un'esperienza che coinvolge la struttura nel suo complesso, in tutte le sue componenti e che può aiutare la famiglia ad avviare un percorso di conoscenza più approfondito sul deficit del figlio.

L'art. 24 della Convenzione ONU chiede anche di procedere ad un'adeguata formazione di coloro che sono impegnati - professionalmente - in tutti i livelli dell'istruzione e dell'educazione; tale formazione dovrebbe «includere la consapevolezza della disabilità e l'utilizzo di appropriate modalità, mezzi, forme e sistemi di comunicazione aumentativi/alternativi, tecniche e materiali didattici adatti alle persone con disabilità». Troppo spesso, però, gli educatori e gli insegnanti mancano della preparazione necessaria a sostenere l'inclusione dei bambini con disabilità nelle scuole e nei servizi educativi per la prima infanzia: tale formazione, in realtà, sarebbe utilissima proprio per l'avvio e il potenziamento dei processi inclusivi ${ }^{17}$.

5. States Parties shall ensure that persons with disabilities are able to access general tertiary education, vocational training, adult education and lifelong learning without discrimination and on an equal basis with others. To this end, States Parties shall ensure that reasonable accommodation is provided to persons with disabilities.

${ }^{16}$ United Nations Educational, Scientific and Cultural Organization, Inclusion of Children with Disabilities: The early childhood imperative, UNESCO Policy Brief on Early Childhood No. 46, UNESCO, Paris, aprile- giugno 2009, Testo disponibile al sito: http://unesdoc.unesco. org/images/0018/001831/183156e.pdf. Consultato il 9 dicembre 2016.

${ }^{17}$ Un esame della situazione dei bambini con disabilità intellettive in 22 paesi europei ha sottolineato come la formazione degli insegnanti si sia dimostrata efficace nel favorire l'inclusione. Secondo uno studio del 2003, i dirigenti scolastici che avevano seguito un maggior numero di corsi sulla disabilità esprimevano punti di vista più inclusivi. E di questo nuovo atteggiamento hanno beneficiato gli studenti: punti di vista positivi sull'inclusione, infatti, si sono tradotti in un minor numero di soluzioni restrittive per gli studenti con disabilità: cfr. Praisner Cindy L. (2003), "Attitudes of Elementary School Principals toward the Inclusion of Students with Disabilities", Exceptional Children, vol. 69, n. 2, pp. 135-145.

Chi era stato formato all'istruzione inclusiva mostrava atteggiamenti più positivi rispetto a chi non aveva ricevuto alcuna formazione; inoltre, a vantare gli atteggiamenti migliori erano 
In Italia, la disciplina dei servizi per l'infanzia risale alla Legge 1044 del $1971^{18}$, istitutiva degli asili nido, che ne ha affidato la programmazione e la regolamentazione alle Regioni e la loro gestione alle Amministrazioni comunali. Ciò ha prodotto una diversificazione crescente delle normative e una distribuzione diseguale degli asili nido sul territorio nazionale, a seconda della diversa capacità economica degli enti locali. Invece, l'intervento progressivo dello Stato ha permesso di accogliere gradualmente nelle scuole per l'infanzia circa il 94\% dei bambini fra 3 e 6 anni.

La Legge 1044 indicava che i nidi dovevano «essere dotati di personale qualificato sufficiente e idoneo a garantire l'assistenza sanitaria e psicopedagogica del bambino», ma nella normativa statale di riferimento e nelle prime leggi applicative a livello regionale non si prevedevano disposizioni specifiche per l'accoglienza di bambini con disabilità. La Legge Quadro n. 104/1992 ${ }^{19}$ sancisce non solo il diritto dei bambini con disabilità alla frequenza della scuola per l'infanzia, ma anche, all'art.12, comma 2, che «è garantito il diritto alla educazione e all'istruzione della persona handicappata nelle sezioni di scuola materna». Questo significa che la priorità di iscrizione alla scuola dell'infanzia materna del bambino con disabilità è un diritto esigibile, e che tale diritto è esteso ai bambini più piccoli relativamente all'accesso agli asili nido. All'art. 3 , comma 3 della Legge 104/92 si legge che «le situazioni riconosciute di gravità determinano priorità nei programmi e negli interventi dei servizi pubblici». Ciò significa che $\mathrm{i}$ bambini con disabilità complessa devono avere diritto di priorità di accesso agli asili nido. Un asilo nido, pertanto, deve accogliere, inserire, integrare e includere ogni bambino, attivando le risorse necessarie e gli strumenti previsti per il sistema dell'istruzione. Nell'art. 12 della legge 104/1992, ai commi 5 e 6, è previsto che l'inserimento dei bambini con disabilità vada definito anche in collaborazione con i servizi appositi delle ASL e con i servizi sociali dei Comuni ${ }^{20}$.

gli insegnanti dotati di un'effettiva esperienza in merito: cfr. De Boer A., Pijl S.J., Minnaert A. (2011), "Regular Primary Schoolteachers Attitudes towards Inclusive Education: A review of the literature", International Journal of Inclusive Education, vol. 15, n. 3, aprile, pp. 345-346).

Tuttavia, la formazione curricolare prepara di rado gli insegnanti a un'istruzione inclusiva. Laddove esiste, questa formazione è di qualità variabile. Sebbene ne esistano in gran numero, corsi e manuali non si rivelano sempre adeguati a un contesto specifico perché contengono spesso concetti astratti e non danno sufficienti strumenti pratici d'insegnamento.

${ }^{18}$ Legge 1044 del 1971. Testo disponibile al sito: https://www.senato.it/japp/bgt/showdoc/17/ DOSSIER/757589/index.html?part=dossier_dossier1-sezione_sezione11-h2_h21. Consultato il 9 dicembre 2016.

19 Legge-quadro per l'assistenza, l'integrazione sociale e $i$ diritti delle persone handicappateTesto disponibile al sito: http://www.gazzettaufficiale.it/eli/id/1992/02/17/092G0108/ sg. Consultato il 9 dicembre 2016.

${ }^{20}$ Nell'asilo nido non è prevista la figura dell'insegnante di sostegno, ma qualora siano presenti bambini con deficit, certificati ai sensi della legge 104/92 e la cui condizione sia stata dichiarata all'atto dell'iscrizione, il nido può inoltrare richiesta di personale aggiunto alla 
Nel 2002, la Corte costituzionale ha riaffermato che «il servizio fornito dall'asilo nido non si riduce ad una funzione di sostegno alle famiglie nella cura dei figli o di mero supporto per facilitare l'accesso dei genitori al lavoro, ma comprende anche finalità formative, essendo rivolto a favorire l'espressione delle potenzialità cognitive, affettive e relazionali del bambino»e, pertanto, «la frequenza dell'asilo [è] un essenziale fattore per il recupero» del bambino disabile ${ }^{21}$.

La legge 5 maggio 2009, n. $42^{22}$, sul federalismo fiscale, ha successivamente riconosciuto i nidi come servizi fondamentali e quindi oggetto di finanziamento da parte della fiscalità generale. Il Piano straordinario per lo sviluppo dei servizi socio-educativi territoriali per la prima infanzia, varato dalla Legge 27 dicembre $2006^{23}$, ha costituito l'occasione più importante - dal 1971 - per rilanciare politiche statali di promozione e sostegno dei servizi per i bambini e le bambine sotto i tre anni, attraverso l'avvio di un processo di collaborazione tra le istituzioni dello Stato, delle Regioni e dei Comuni e di definizione dei livelli essenziali per la concreta attuazione dei diritti dei bambini e delle bambine ${ }^{24}$.

L'offerta pubblica di servizi socio-educativi per la prima infanzia si caratterizza per enormi differenze territoriali, sia in termini di spesa che di utenti. Si conferma la carenza di strutture nelle regioni del Sud e non sono visibili segnali di convergenza; aumenta la distanza fra le Regioni in cui il sistema di servizi per la prima infanzia è più consolidato e le Regioni in cui l'offerta pubblica è tradizionalmente più carente ${ }^{25}$.

Commissione $\mathrm{H}$ del Comune che provvederà all'assegnazione di un educatore in appoggio alla sezione, con un rapporto educatore/alunni con necessità di sostegno di 1:2 di massima. Il Piano Educativo Individualizzato (PEI) è un obbligo di legge esteso all'asilo nido. In conformità con la legge nazionale, i regolamenti dei Nidi d'Infanzia prevedono che i bambini con disabilità abbiano la precedenza.

${ }^{21}$ Corte Costituzionale, sentenza n. 467 / 2002. Testo disponibile al sito: http://www. cortecostituzionale.it/actionSchedaPronuncia.do?anno=2002\&numero=467 9 dicembre 2016.

${ }^{22}$ Testo disponibile al sito: http://www.parlamento.it/parlam/leggi/09042l.htm. Consultato il 9 dicembre 2016.

${ }^{23}$ Testo disponibile al sito: http://www.parlamento.it/parlam/leggi/062961.htm. Consultato il 9 dicembre 2016.

${ }^{24}$ Nell'anno scolastico 2012/2013, l'1,2\% dei bambini tra zero e due anni (circa 20 mila) ha usufruito dei servizi integrativi per la prima infanzia. Tale quota risulta in diminuzione rispetto ai tre anni precedenti; il 79,3\% dei bambini di 0-2 anni non usufruisce di alcun servizio socioeducativo per la prima infanzia http://www.istat.it/it/archivio/offerta+asili+nido. Consultato il 9 dicembre 2016.

${ }^{25}$ I bambini che usufruiscono di asili nido comunali o finanziati dai comuni variano dal $3,6 \%$ dei residenti fra 0 e 2 anni al Sud al 17,5\% al Centro. La percentuale dei Comuni che garantiscono la presenza del servizio varia dal $22,5 \%$ al Sud al $76,3 \%$ al Nord-est. Nella distribuzione regionale dell'indicatore di presa in carico degli utenti per l'anno 2012/2013, ai due estremi vi sono la Calabria, con il 2,1\% (in calo dal 2,5\% dell'anno precedente) e l'Emilia- 
A nostro avviso, sarebbe molto importante migliorare l'educazione precoce dei bambini e delle bambine con disabilità; a tal fine, il Gruppo di lavoro n. 4 dell'OND - che la scrivente ha co-coordinato - ha individuato alcuni miglioramenti possibili nella Legge 107/2015 ${ }^{26}$ di Riforma del sistema nazionale di istruzione e formazione e delega per il riordino delle disposizioni legislative vigenti: tali indicazioni saranno diffuse dopo la pubblicazione ufficiale del II Programma di Azione biennale per la promozione dei diritti e l'integrazione delle persone con disabilità.

\section{Professioni di aiuto, cura educativa e elementi genitoriali}

Canevaro indica come professioni d'aiuto quelle che entrano in rapporto con un/una bambino/a in un momento particolare del loro percorso (ad esempio, quando i bambini frequentano l'asilo nido, la scuola dell'infanzia ecc.), permettendo di sviluppare la propria crescita e la propria vita per tutto l'arco della stessa. Tuttavia, vi sono delle circostanze particolari che riguardano momenti specifici o situazioni complesse e che esigono delle attenzioni peculiari: sono queste occasioni, soprattutto, che chiamano in causa le professioni d'aiuto e che sembrano decisamente legate al binomio educazionesalute per tutto l'arco della vita. Come molti studiosi sostengono, il quadro delle professioni d'aiuto è, in questo momento storico, alquanto nebuloso e incerto e necessiterebbe di un completo riesame nel quale individuare e ri-precisare $\mathrm{i}$ percorsi formativi e le affinità tra una professione e l'altra (ruolo dell'educatore e ruolo dell'insegnante, ad esempio): è utile, allora, provare a puntualizzarne qualche elemento basilare.

Fondamentale è che il lavoro di cura, educativamente inteso, e la relazione d'aiuto non si confondano con il loro stesso fine (che è anche quello di permettere al minore disabile di vivere in mezzo agli altri), e non si irrigidiscano in interminabili iniziative tese a rafforzare il curare, in senso medico e psichiatrico, e, parallelamente, dirette a cancellare l'esistenza della persona e il suo progetto di vita: così facendo, infatti, la relazione d'aiuto diviene un'imposizione di aiuto asettica e impersonale, priva della dimensione dialogica della relazionalità e mancante di quello scambio di umanità che rende significative le relazioni tra le persone. Le professioni d'aiuto si espletano anche nella consapevolezza che le persone che stanno con un disabile hanno bisogno di fare e sarebbe una

Romagna, con il 27,3\% (in lieve aumento dal 27,2\% dell'anno precedente) http://www.istat.it/it/ archivio/offerta+asili+nido. Consultato il 9 dicembre 2016.

${ }^{26}$ Testo disponibile al sito: http://www.gazzettaufficiale.it/eli/id/2015/07/15/15G00122/sg. Consultato il 9 dicembre 2016. 
buona qualità professionale degli operatori fornire questo qualcosa da fare per evitare la ricerca cieca e insulsa o la ricerca esasperata di iniziative e attività da avviare (Canevaro, 2006, pp. 48-49).

Il fatto di non avere tutto, di conoscere limiti e sofferenza, non è motivo per rinunciare a vivere in pienezza e come tutti coloro che vivono a piene mani la vita, a fare e ad essere. Anche quando il futuro appare inesistente o vuoto, ciascun genitore, ad esempio, che si trova in situazione di bisogno, deve individuare l'inizio di una risposta, anche se (anzi, forse proprio in forza di questo avvenimento) ciò avviene nell'esperienza del dolore, della privazione affettiva, della limitazione fisica. Le professione di aiuto vengono a contatto, molto più frequentemente di altre, con il dolore, con limiti abnormi, con vincoli prepotenti: tale esperienza è spesso logorante, problematica, non solo per i bambini disabili e i loro genitori, ma per lo stesso educatore (o educatrice) che sperimenta, quotidianamente, esperienze psichiche quali la frustrazione, i meccanismi di difesa. Si tratta di una professione difficile, ma proprio nell'esperienza del limite vi può essere un'autentica riflessione critica, di affinamento di sé, di ri-apertura di impreviste possibilità: nell'esperienza del dolore e del limite, l'educatore (o l'educatrice) dell'asilo-nido, che è impegnato con un bambino con disabilità, interviene allo scopo di ridurre l'handicap, ad esempio, motivando il recupero dei desideri, perché la malattia e la disabilità inchiodano il soggetto, limitandone le dinamiche relazionali, nel senso che producono una distanza tra desiderio ed effettive possibilità di realizzazione.

Il presente e il futuro dell'identità delle professioni educative di aiuto dipendono dal carattere etico della scommessa educativa, perché l'educatore problematizza situazioni schematizzate e classificate, ricollocandole in un contesto significativo di potenzialità, trasformando le limitazioni esistenti in possibilità e in orizzonti di senso: si tratta di un lavoro utopico, intendendo l'utopia come ideale regolativo, di traino, che cerca di travalicare il limite.

Come spiega Conte (Conte, in Antinori, a cura di, 2000 p. 17), nell'interpretazione data da Heidegger, l'Esserci assume il suo significato proprio in relazione alla cura. Dal punto di vista ontologico, l'essere nel mondo dell'uomo non può essere compreso se non in riferimento alla cura. Essa è qualcosa che esiste prima di ogni "comportamento" e di ogni "situazione" dell'Esserci, in quanto ogni momento della vita è determinato "dalla Cura e della Cura porta inevitabilmente il segno" (ibidem, p. 19). Essa designa un rapporto di prossimità e di incontro sia con le cose che sono presenti nel mondo sia con altre persone che lo popolano. Questo mondo in cui si attua la relazione è un mondo comune già dato che ci occupa ancor prima che il singolo uomo inizi ad occuparsi di lui. Tra le modalità che il filosofo tedesco espone dell'essere nel mondo ne distingue due: il prendersi cura e l'aver cura. Col primo egli intende la relazione con gli oggetti mentre col secondo si designa la relazione 
con gli altri esseri umani. Solamente nell'aver cura noi incontriamo l'altro Esserci, che viene riconosciuto in quanto soggetto. Esistono due modalità estreme dell'aver cura che l'uomo deve conoscere se vuole giungere ad una relazionalità autentica. La prima modalità pone l'altro "fuori”" dalla relazione; ci si sostituisce ad esso, lo si solleva dalla cura, si fa e si decide per lui. L'altro diviene in tal caso o un dipendente o un dominato. In ogni caso la relazione è soffocata. Nell'aver cura in senso autentico, invece, si aiuta l'altro ad essere consapevole e libero per la propria cura, lo si rispetta e ci si relaziona con lui. Nel primo caso ci si sostituisce all'altro e lo si domina; in quest'ultimo, lo si anticipa liberandolo: ed è in quest'ultima direzione che rientra anche l'indicazione di Mahoney a "farsi guidare dal bambino" nell'intervento educativo (Mahoney, in Rondal, Rasore-Quartino A. a cura di, 2007, pp. 90-106). La Cura ci mostra il senso formativo dell'agire umano il quale, però, è costantemente esposto al rischio di far ricadere l'altro in una relazione inautentica e dannosa. Si è visto come, purtroppo, la storia dei disabili sia una un susseguirsi di normalità negate, che costringe le persone con disabilità ad una vita inautentica e lontana dalla società; costrette entro il ruolo marginale che è stato costruito attorno al loro deficit esse saranno viste come perennemente bisognose delle cure preconfezionate da altri.

Per superare questa visione riduttiva ed estremamente dannosa occorre che genitori ed educatori attuino la cura autentica, in cui il bambino e la bambina vengono riconosciuti come persone e non come "sottopersone" imprigionate in una perenne dipendenza. Aver cura di bambini e bambine con disabilità significa aiutarli a realizzare responsabilmente la cura di sé, senza sostituirsi ad essa, senza intromettersi (Caldin, 2007, p. 90; Caldin, 2013, 2014; Caldin, Serra, a cura di, 2011; Visentin, Caldin, Chiandetti, 2015): in tal senso, la relazione educativa diviene il punto in cui si attua il cambiamento, in cui si restituisce all'altro la normalità negata.

Chi svolge un lavoro educativo deve avere una grande chiarezza verso se stesso: il lavoro con i bambini molto piccoli e con i loro genitori si accompagna, infatti, all'individuazione delle proprie risorse e potenzialità, ma anche dei propri limiti e delle proprie difficoltà. Perciò, l'educatore che lavora con i piccolissimi dovrebbe possedere una buona maturità psicologica che lo aiuti ad implementare le capacità personali, ma anche ad accettare le proprie inadeguatezze, e una sufficiente capacità di leggere il proprio sé nel contesto reale, costituito, oltreché dai minori e dalle loro famiglie, anche dagli altri educatori e/o dal personale ausiliario. In quest'ottica, divengono rilevanti le conoscenze che alcune discipline offrono agli educatori: in modo particolare, quelle provenienti dalla pedagogia, dalla psicologia e dalla psicoanalisi possono prospettare delle coordinate importanti per il problema dell'igiene mentale di chi esercita una professione educativa. Le professioni educative, 
infatti, più di altre, vengono a contatto, frequentemente, con due esperienze psichiche rilevanti: la frustrazione e i meccanismi di difesa. La prima risulta alquanto abituale nell'educare, poiché i tempi sono estremamente dilazionati e gli obiettivi, talvolta, raggiunti solo parzialmente; l'educatore, poco consapevole degli effetti di tali frustrazioni, può confondere gli insuccessi contingenti con l'incapacità personale e accrescere la disistima e la svalutazione di sé. I meccanismi di difesa, che assolvono ad un ruolo vitale di protezione e di salvaguardia, possono diventare abnormi quando le situazioni risultano particolarmente conflittuali e frustranti. Così, per esempio, quando non si riesce a fronteggiare un'occasione professionale complessa e gravosa (l'ambientamento particolarmente difficile di un bambino disabile piccolissimo; il distacco, molto problematico, di un bambino dai suoi genitori; la relazione educativa con un bambino con disabilità complessa ecc.), la si può privare di significatività attraverso un'estrema razionalizzazione o con l'uso sistematico della proiezione che induce ad attribuire ad altri l'insuccesso dei propri errori. In queste circostanze, una sufficiente conoscenza e una buona consapevolezza dei meccanismi di difesa possono guidare ad un loro veloce riconoscimento, mettendo l'educatore in grado di attivare strategie di coping o di limitazione degli stessi. A questi accorgimenti nell'esercitare la professione educativa, proficui per la salute psichica degli educatori - ma anche per quella dei bambini e dei loro genitori - si aggiunge quello di lavorare in équipe, o comunque non in solitudine, per promuovere e incrementare il confronto, la discussione, per attenuare il senso di onnipotenza e quello di coltivare una significativa, variegata e soddisfacente vita privata che aiuti a sdrammatizzare i contrasti e i dissensi delle contingenze professionali, senza che ne siano sviliti l'impegno e la tensione progettuale (Bertolini, 19887, pp. 260-262).

Nella relazione con il genitore del bambino piccolo (come in altre situazioni educative), frequentemente, l'educatore esplica un'importante funzione che da una parte si connota come promozionale (di progetti, di iniziative, di proposte, di decisioni ecc.) e, dall'altra, come contenitiva (dell'ansia di separazione, dei sensi di colpa che possono derivare dall'essere costretti a lasciare i propri figli ad altri ecc.). Educatori capaci di promozione e di contenimento possono risultare figure essenziali per non ingigantire la solitudine educativa genitoriale e per incoraggiare e condividere quei percorsi di autonomia e di emancipazione dei figli che, talvolta, risultano problematici. Nel lavoro tra gli educatori e le famiglie, infatti, si verifica una situazione in cui, a volte, la relazione è simmetrica (educatori e genitori discutono insieme e insieme si attivano per il minore), altre volte il genitore è oggetto stesso dell'intervento quando, ad esempio, gli educatori intervengono per migliorare le sue modalità educative (nei colloqui individuali, nelle assemblee ecc.).

Negli educatori che lavorano con bambini piccoli diviene chiaro, allora, un elemento della relazione educativa, ossia la presenza di elementi genitoriali: 
gli educatori, infatti, nel loro lavoro, affrontano esperienze emotive e difficoltà molto simili a quelle delle figure genitoriali e, tuttavia, la loro situazione è molto diversa non solo per il contesto nel quale operano (un setting maggiormente protetto e transitorio), ma anche per l'assenza di sensi di colpa che possono - invece - avere i genitori, quando sono costretti a trascorrere molto tempo lontano dai loro figli. Anche l'educatore che lavora nei servizi per la prima infanzia, infatti, deve contemperare socializzazione e apprendimento, svolgendo due funzioni care a W.R. Bion, di cui anche F. Fornari ha ampiamente trattato (Bion, 1972; 1971; Fornari, 1976, 1981). Secondo tale orientamento, che getta le sue fondamenta nelle teorie psicoanalitiche e gruppali, le relazioni intrafamiliari si esplicano attraverso modalità protettive e emancipative, le prime volte all'accoglimento del bisogno e del desiderio del bambino e alla loro traduzione, le seconde intese come richieste di sforzo graduate secondo le capacità del soggetto in crescita. Entrambe le modalità educative, sia quelle ascritte alla protezione, all'accoglimento e al sostegno che quelle tese alla separazione, all'autonomia e alla frustrazione, dovrebbero essere comprese nei differenti codici familiari, mantenendo una declinazione integrata e non scissa salvaguardando, così, sia la sopravvivenza del bambino reale che ha bisogno, innanzitutto, di protezione, di aiuto, di accudimento e di difesa, ma anche del bambino culturale che, per diventare tale, proprio di differenziazione, di autonomia, di separazione e di prove ha bisogno (Fornari, Frontori, Riva Crugnola, 1985). Anche gli educatori possono promuovere crescita personale (cognitiva, affettiva e relazionale), attraverso l'utilizzo di un approccio protettivo (orientato all'accoglimento del bisogno, alla soddisfazione delle richieste ecc.) ed emancipativo, teso a sollecitare i primi incontri alle norme comunitarie, al rispetto dell'altro ecc.

\section{L'educazione inclusiva nell'asilo nido}

La prospettiva inclusiva richiama l'ICF dell'OMS (WHO 2001; OMS, 2002), indicando che un contesto educativo facilitante - ad esempio asilinido accoglienti e integranti - non potrà mai ridurre il deficit (la variabile irreversibile, oggettivabile), ma aiuterà a contenere gli elementi che vanno ad aggravarlo, cioè quelli handicappanti. La classificazione dell'OMS guarda con estrema attenzione all'interazione tra il bambino e il suo ambiente (fatto di persone, di relazioni, di occasioni, di opportunità); tale incontro tra un bambino, con le sue caratteristiche - quindi anche con un eventuale deficit - e i fattori contestuali può produrre un handicap (barriera, pregiudizio, mancanza di scuole/asili-nido). In tal senso, lavorare per l'inclusione significa anche lavorare per la riduzione dell'handicap, cercando di individuare le variabili 
in gioco nell'incontro tra un bambino con deficit e il contesto, per chiarire quali possano costituire un ostacolo al pieno sviluppo delle potenzialità del bambino e quali, invece, lo facilitino: questo rimane uno dei compiti prioritari dell'educazione e della pedagogia speciale.

L'elemento ricorrente dei contesti educativi facilitanti ci riconduce anche al concetto di inclusione. Come sappiamo, in ambito internazionale vi è un' evoluzione del termine integrazione in inclusione, caratterizzata da una forte centratura sul contesto, e non solo sul singolo bambino. L'educazione inclusiva invita a considerare non solo il bambino con disabilità, ma i compagni stessi che possono produrre integrazione/inclusione, considerando anche il problema della gestione dei tempi dei bambini, con un'attenzione sia a rallentare i tempi (se necessario), ma anche a spingere i tempi (come a volte fanno i bambini tra loro). E la sollecitazione è tanto più incisiva quanto più effettuata dai compagni che rendono paritaria e reciproca la relazione interpersonale anche in situazioni problematiche. L'educazione inclusiva contempla la dimensione del lavoro di rete e si rifà ad un modello complesso di disabilità che sia occasione di conoscenza e non solo di socializzazione e nel quale si costruiscano reti di integrazione che potenzino i processi di apprendimento, che facilitino la familiarizzazione, intesa come conoscenza diretta dei bambini con disabilità, e che permetta l'attenuazione degli stereotipi e l'evoluzione delle rappresentazioni personali e sociali (anche da parte dei compagni) sulla disabilità.

Quando in una famiglia arriva un bambino con disabilità, la dimensione temporale, già usualmente sconvolta dall'arrivo di un figlio, soprattutto se primogenito, viene ad avere insolite connotazioni. Innanzitutto, il tempo dovrebbe diventare occasione per avviare almeno alcuni processi di elaborazione del lutto del bambino atteso: è un tempo "della" e "per la" madre (del/per il padre), ma anche "del" e "per il" bambino. Quest'ultimo, infatti, necessita delle attenzioni della madre, ma anche della sua distanziazione: di un processo, cioè che permetta alla madre di avere dei pensieri sul bambino, ma in uno spazio-tempo lontano dal bambino stesso. Simultaneamente, questo diviene anche il tempo in cui il bambino può elaborare l'assenza/separazione della madre, in maniera graduale e non traumatica. È ' infatti questa che permette, anche al bambino con disabilità, di avanzare (con i suoi tempi) verso la "nascita psicologica": l'assunzione di una identità personale - pur con i vincoli che un deficit può imporre - necessita anche dell'esperienza di frustrazione che il bambino deve conoscere.

L'asilo-nido può connotarsi come un servizio di sostegno e di accompagnamento ai genitori che hanno un bambino con disabilità: l'inserimento graduale, pianificato, "accompagnato" da educatori preparati e attenti, in grado di "incontrare" e di tollerare, per superarli, i vissuti di morte dei genitori (morte delle aspettative, morte di potenziali ruoli futuri ecc.) 
può favorire l'avvio di processi di elaborazione del lutto da parte delle figure parentali e una positiva e contenuta distanziazione del bambino dalle stesse.

Tuttavia, affinché l'integrazione del bambino con disabilità all'asilo nido possa costituire un'occasione di co-evoluzione per tutti (bambini, genitori, educatori) - e divenire processo inclusivo - deve:

- fondarsi su una sufficiente relazione oggettuale tra madre e bambino (senza precocismi e/o forzature inutili) e sul riconoscimento di una figura emancipativa che, procedendo tra spinte emancipative e protettive, favorisca i processi di separazione e di costruzione dell'identità personale;

- contare sulla presenza di personale adeguato e competente;

- configurarsi come un intervento educativo che ha "cura" di tutti e due i genitori, incrementando e sostenendo le capacità parentali generali (un bambino disabile è, innanzitutto, un bambino) e ampliando e supportando anche le risposte ai bisogni specifici, derivanti dalla presenza di un deficit.

Uno dei pregi dei percorsi verso l'inclusione di tutti i bambini è la messa in comune di esperienze e competenze che permette di individuare delle costanti educative che orientano e sollecitano la prospettiva inclusiva. Gli elementi ricorrenti individuati aiutano a codificare alcune buone prassi documentate e a delineare alcune linee teoriche che contribuiscono al rinnovamento delle dimensioni concettuali. Anna Freud - psicoanalista, ma anche maestra d'asilo che ha a lungo esercitato la professione - nel 1930, nelle sue - ancora attualissime! - Conferenze per insegnanti e genitori, scriveva che coloro che lavorano praticamente con i bambini (come molti educatori/educatrici/ insegnanti) vedono e conoscono ogni giorno già molto di essi, ma per mettere ordine in tutto quello che vedono e che conoscono, gli insegnanti/educatori hanno bisogno di fermarsi, di riflettere, di ascoltare, di scrivere (Freud (1930), 1978, pp. 87-131); infatti, quando ci si ferma, riflettendo e documentando il proprio e altrui lavoro, si sollecitano processi di integrazione, svolgendo un'azione che spinge la teoria a generare altre pratiche e ad essere, dalle stesse pratiche, confermata o disconfermata.

Per gli educatori e le educatrici, si verifica un problema di giusta distanza educativa ed emotiva; può succedere, ad esempio, che gli educatori manifestino atteggiamenti relazionali troppo centrati sui propri meccanismi di difesa piuttosto che sulle esigenze educative del bambino; oppure, all'opposto, che focalizzino il totale interesse sulla vita affettiva del soggetto con cui lavorano, progettando e realizzando interventi inadeguati (troppo centrati sulla dimensione affettivo-genitoriale) che risultano in competizione con la famiglia. Come ha indicato Elena Riva, il conflitto interno (ossia, la colpevolizzazione per la quotidiana separazione dal figlio), spesso inconscio e contraddittorio con la scelta, razionale, di far frequentare al figlio un servizio prescolare, può determinare nei genitori, e soprattutto nelle madri, che «... soffrano di 
un'intensa gelosia nei confronti di chi si occupa quotidianamente del loro bambino, e provino sentimenti di esclusione e qualche volta di invidia nei confronti del rapporto che il figlio intrattiene con altre figure femminilimaterne, in particolare se con funzioni di caregiver come le educatrici, nei confronti delle quali replicano e drammatizzano la sfida inconscia con la propria madre» (Riva, 1997, pp. 44-46).

All'interno della problematica della giusta distanza educativa si ritrovano anche i dilemmi sull'adozione e sull'uso di alcune strategie educative; ad esempio, la comprensione degli esiti negativi della critica e del diniego può trascinare l'educatore a utilizzare in maniera esagerata gratificazioni e apprezzamenti: ma questa scelta non esonera da altri errori educativi, dato che la relazione educativa non è positiva, né ottimale, se interamente compiacente. Lo strumento della critica, sempre ponderato, può essere utilizzato in forma pungolante, provocatoria e propositiva anziché in forma sminuente e inibente. Anche l'uso gratuito e sistematico della lode può essere controindicato se viene calibrata esclusivamente sulle azioni, trascurando quello che il bambino è, indipendentemente dal suo successo; così l'elogio ingiustificato e insistente può favorire una possibile dipendenza dall'approvazione e dalla condiscendenza. Va ricordato, però, che più dei singoli interventi ciò che li rende costruttivi o inopportuni è il sentimento che li anima: in questo senso la critica costruttiva è uno strumento importante nella relazione educativa con i piccolissimi, perché il suo uso conferma l'impossibilità di proteggere infinitamente e aiuta ad evitare il rischio di voler esonerare, a tutti costi, dalle situazioni spiacevoli.

Per l'educatore si tratta di conquistare una nuova spontaneità, una spontaneità competente che solleciti a prestare attenzione al proprio modo di sentire nel momento in cui si incontra la vita affettiva dell'altro; una spontaneità competente che non si declina solo sulla conoscenza dei problemi affettivi, ma che si trasforma e si evolve fino a divenire stile di comportamento, esercitato nella consuetudine, rafforzato nell'apprendimento, rinvigorito nell'esercizio, vivificato nella ricerca personale, rinnovato nella possibilità di saper rispondere agli avvenimenti problematici della realtà. Una competenza che concilia affettività e razionalità «un lento procedere a cerchi concentrici equidistanti e tra di loro in equilibrio», «un avanzare armonioso tra capire ed essere, tra consapevolezze ed espressione, senza perfezionismi unilaterali» (Montuschi, 1993, pp. 182-193; 1997; Montuschi e Caldin, coordinamento di, 2004).

\section{Riferimenti bibliografici}

Bertolini P. (19887). L'esistere pedagogico. ragioni e limiti di una pedagogia come scienza fenomenologicamente fondata. Firenze: La Nuova Italia. 
Bion W.R. (1971). Esperienze nei gruppi. Roma: Armando.

Bion W.R. (1972), Apprendere dall'esperienza. Roma; Armando,

Booth T. e Ainscow M. (2002). Index for inclusion: developing learning and partecipation in schools, Bristol: CSIE.

Caldin R. (2014). Inclusive social networks and inclusive schools for disabled children of migrant families, ALTER, 8, pp. 105-117. DOI: 10.1016/j.alter.2014.02.001

Caldin R. (2013). Educability and possibility, difference and diversity: the contribution on Special Pedagogy, Education sciences \& society, 2, pp. 65-77.

Caldin R. (2007). Introduzione alla pedagogia speciale. Padova: Cleup.

Caldin R., Serra F., a cura di (2011). Famiglie e bambini/e con disabilità complessa. Comunicazione della diagnosi, forme di sostegno, sistema integrato dei servizi. Padova: Fondazione Zancanevaro A. (2006). Le logiche del confine e del sentiero. Trento: Erickson.

Conte M. (2000). La "cura": tema esistenziale e competenza pedagogica, in Antinori F. (a cura di), Disagio, lavoro di cura e relazione d'aiuto. Padova: Cleup

De Boer A., Pijl S.J., Minnaert A. (2011). Regular Primary Schoolteachers Attitudes towards Inclusive Education: A review of the literature, International Journal of Inclusive Education, vol. 15, pp. 345-346.

Fornari F. (1976) Simbolo e codice. Milano: Feltrinelli.

Fornari F. (1981). Il codice vivente, Torino: Bollati Boringhieri.

Fornari F., Frontori L., Riva Crugnola C. (1985), Psicoanalisi in ospedale. Nascita e affetti nell'istituzione. Milano: R. Cortina.

Freud A. (1930). Quattro Conferenze di psicoanalisi per insegnanti e genitori. In Freud A. (1978). Opere, Vol. I, Bollati Torino: Boringhieri, pp. 87-131.

Mahoney G. (2007). Cognitive rehabilitation, in Rondal J.A., Rasore-Quartino A. (a cura di). Therapies and rehabilitation in Down syndrome, pp. 90-106, xiv, 214 pp., New York, NY, US: John Wiley \& Sons Ltd; US

Montuschi F. (1993). Competenza affettiva e apprendimento. Dalla alfabetizzazione affettiva alla pedagogia speciale. Brescia: La Scuola..

Montuschi F. (1997) Fare ed essere. Il prezzo della gratuità nell'educazione. Assisi: Cittadella.

Montuschi, R. Caldin, a cura di (2004), Disabilità, integrazione e pedagogia speciale, Padova: Cedam, numero monografico della rivista Studium Educationis, n.3.

Praisner Cindy L. (2003). Attitudes of Elementary School Principals toward the Inclusion of Students with Disabilities, Exceptional Children, vol. 69, n. 2, pp. 135-145.

Riva E. (1997). Figli a scuola. Milano: FrancoAngeli.

UNESCO (2005). Guidelines for Inclusion: Ensuring Access to Education for All, Paris, Unesco.

UNESCO (2006). Positive discipline in the incluisive, learning-friendly classroom: a guide for teachers and teacher educators, Bangkok: Unesco.

UNESCO (2010). Education for all, Unesco.

United Nations Educational, Scientific and Cultural Organization (2009), 'Inclusion of Children with Disabilities: The early childhood imperative', UNESCO Policy Brief on Early Childhood No. 46, Paris: UNESCO.

Visentin S., Caldin R., Chiandetti L. (2015), Families with Pluridisabled Children: The Parental Point of View on their Relationship with Health and Social Services, in: Visentin S., Caldin R., Chiandetti L., Innovative Practice and Interventions 
for Children and Adolescents with Psychosocial Difficulties and Disabilities, Newcastle: Cambridge Scholars, pp. 92-112.

WHO (World Health Organization, 2001). ICF: International Classification of Functioning, disability and health, Geneva; trad. it. OMS (Organizzazione Mondiale della Sanità, 2002), ICF: Classificazione Internazionale del funzionamento, della disabilità e della salute. Trento: Erickson.

WHO, The World Bank, World Report on Disability, Geneva, 2011 http://whqlibdoc. who.int/publications/2011/9789240685215_eng.pdf.

Winzer M., Mazurek K. (2000). Special education in the 21st-century. Issues of inclusion and reform, Washington Gallaudet University Press. 